\title{
Semiclassical theory for absorption of radiation in particles with diffusive electron motion
}

\author{
Bernhard Mehlig† and Michael Wilkinson $\ddagger$ \\ Institute for Theoretical Physics, University of California at Santa Barbara, Santa Barbara, CA \\ 93106, USA
}

Received 9 September 1996, in final form 20 December 1996

\begin{abstract}
We present a semiclassical analysis for the electric dipole absorption of electromagnetic radiation by small metallic particles, in the case where the electron motion is diffusive. We find that there are two contributions to the one-body effective potential: the 'static' potential makes a dominant contribution to the absorption coefficient below the frequency $\omega_{c}=D / a^{2}$, and the 'dynamic' potential is dominant for frequencies $\omega \gg \omega_{c}$ (here $D$ is the diffusion constant, and $a$ the size of the particle). Remarkably, these two contributions sum to give exactly the classical absorption coefficient for all frequencies.
\end{abstract}

\section{Introduction}

The absorption of electromagnetic radiation by a dilute dispersion of small metallic particles can be analysed using classical electrodynamics by using an effective-medium theory to evaluate the imaginary part of the dielectric constant [1-3]. The corresponding quantum theory, reviewed in [4-6], has attracted considerable interest, largely stimulated by a suggestion by Gorkov and Eliashberg [7] that the absorption coefficient would be reduced when the photon energy $\hbar \omega$ is made smaller than the mean spacing $\Delta$ of single-particle levels; measurement of the absorption coefficient in this frequency range would give an experimental verification that the statistics of the energy levels is in agreement with randommatrix theory [8]. However, for frequencies such that $\omega \gg \omega_{0} \equiv \Delta / \hbar$, the absorption coefficient predicted by Gorkov and Eliashberg is much larger than that predicted by classical electrodynamics, because they neglected the screening of the externally applied electric field by the polarization charge induced on the surface of the particle [9]. The same error occurs in many other published calculations of the quantum theory of absorption by small particles. Calculations taking account of screening have been discussed by several authors using a variety of approaches [10-16]; we will review these contributions later. In this paper we give a transparent analytical treatment of this problem, for the case where the electron motion is diffusive, and verify its correctness by showing its correspondence with the predictions of classical electrodynamics. The corresponding analysis for the case of ballistic electron dynamics is given in $[17,18]$.

Our analysis is confined to frequencies far below the plasma frequency $\omega_{p}$, and we assume that the size (for circular particles, radius) $a$ of the particle is small compared to the

$\dagger$ Permanent address: Max-Planck-Institut für Physik komplexer Systeme, Bayreuther Strasse 40, Haus 16, 01187 Dresden, Germany.

$\ddagger$ Permanent address: Department of Physics and Applied Physics, John Anderson Building, University of Strathclyde, Glasgow G4 0NG, UK. 
wavelength of the radiation, so that the spatial dependence of the externally applied fields can be neglected. The motion of electrons within the particle is assumed to be diffusive, so that it can be characterized by a diffusion constant $D$ which is proportional to the DC bulk conductivity $\sigma_{0}$ of the metal. The semiclassical approximations are justified if the size of the particle is large compared with the Fermi wavelength, and if the photon energy is large compared to the level spacing. We also assume that the size of the particles is very small compared to the electromagnetic skin depth $\delta$.

The response of a finite-sized quantum system to an external perturbation $\widehat{U}$ is characterized by the matrix elements of the perturbation, in the basis formed by the eigenfunctions $\left|\psi_{n}\right\rangle$ of the unperturbed Hamiltonian $\widehat{H}_{0}$, with energies $E_{n}$. These matrix elements $U_{n m}=\left\langle\psi_{n}|\widehat{U}| \psi_{m}\right\rangle$ usually become very small when $\left|E_{n}-E_{m}\right|$ becomes large compared to an energy $E_{c}$ which is related to the time-scale $\tau_{c}$ for decay of the correlation function of $U$ by $E_{c} \tau_{c}=\hbar$. Thouless [19] discussed the significance of this correlation energy scale for the conductance of finite-sized systems in which the electron motion is diffusive; the relevant energy scale is $E_{c}=\hbar \omega_{c}$, where $\omega_{c}=D / a^{2}$. In this context $E_{c}$ is usually termed the Thouless energy. It might be anticipated that the absorption coefficient would behave differently for photon energies above and below the Thouless energy, and if screening were neglected, this would certainly be the case. A surprising feature of our calculation is that the absorption coefficient takes exactly the same form on either side of the Thouless energy.

Our calculation assumes that the electrons move independently in an effective onebody potential, in which the effects of the electron-electron interactions are included self-consistently; we will use a Thomas-Fermi approximation, but it can easily be extended. The externally applied electric field $\mathcal{E}=\operatorname{Re}\left[\mathcal{E}_{0} \exp (\mathrm{i} \omega t)\right]$ produces a perturbation $\operatorname{Re}\left[U_{\text {eff }}(\boldsymbol{r}, \omega) \exp (\mathrm{i} \omega t)\right]$ of the effective one-body potential; we will write

$$
U_{\text {eff }}=U_{\text {stat }}+U_{\text {dyn }}
$$

where the two contributions to the perturbation of the effective potential take the form

$\operatorname{Re}\left[U_{\text {stat }}(\boldsymbol{r}) \exp (\mathrm{i} \omega t)\right]=v_{\text {stat }}(\boldsymbol{r}) \mathcal{E} \quad \operatorname{Re}\left[U_{\mathrm{dyn}}(\boldsymbol{r}, \omega) \exp (\mathrm{i} \omega t)\right]=v_{\mathrm{dyn}}(\boldsymbol{r}) \frac{\mathrm{d} \mathcal{E}}{\mathrm{d} t}$

where the functions $v_{\text {stat }}(\boldsymbol{r})$ and $v_{\mathrm{dyn}}(\boldsymbol{r})$ are independent of time, and will be determined later. The 'static' potential $U_{\text {stat }}$ is that produced by a static externally applied field. It will be determined by the requirement that it produces a screening charge distribution which exactly cancels the externally applied field within the interior of the particle. The second term in (1.1) (the 'dynamical' potential) will be determined by the requirement that it should cause the electron density to rearrange sufficiently rapidly that the screening charge is maintained as the external potential is varied. We find that for frequencies large compared to $\omega_{c}$, the contribution to the absorption coefficient from the dynamical potential $U_{\mathrm{dyn}}$ is dominant, and that this exactly reproduces the classical absorption coefficient. This result would be expected on physical grounds. On the other hand, at frequencies small compared to $\omega_{c}$, the contribution from the static potential $U_{\text {stat }}$ is dominant. We find that this also reproduces the classical result exactly, and that the sum of the two contributions is exactly equal to the classical expression for all frequencies satisfying $\omega_{0} \ll \omega \ll \omega_{p}$.

Before describing our calculation in detail we will briefly discuss some of the papers which have considered the effects of screening in quantum mechanical calculations of the absorption coefficient. Strässler, Rice and Wyder [9] pointed out that screening is important, and a later paper by Rice et al [10] discussed the calculation of the static polarizability using a Thomas-Fermi approximation. The Thomas-Fermi approach was applied to the absorption of radiation in particles with ballistic electron motion by Austin and Wilkinson [17, 18], and 
in the case of diffusive motion by Efetov [11]. The paper by Efetov does not incorporate the additional dynamical potential which is necessary to reproduce the classical behaviour of the system in the high-frequency limit (in the case of ballistic electron motion, the dynamical potential is not required). Moreover, it does not indicate how analytical expressions for the screening potential can be obtained. Sivan and Imry [12] have discussed the effective conductivity of finite-sized samples, and have used effective-medium theory to discuss absorption by small conducting spheres. They reported that the absorption coefficient is the same above and below the Thouless frequency $\omega_{c}$, but their published calculation does not explain why the dimensionless prefactor should be the same in both regimes. Their approach is also subject to the criticism that the effective-medium approach assumes that the spheres are described by a dielectric constant defined for a bulk medium, and the justification for using a size-dependent effective conductivity or dielectric constant is not discussed. Sivan and Imry have pointed out another significant shortcoming of Gorkov and Eliashberg's theory; they showed that the frequency dependence of the absorption coefficient, predicted by a correct application of linear response theory in the limit of zero temperature, is $\alpha(\omega) \sim \omega^{\beta+1}$ for $\hbar \omega \ll \Delta$, rather than the $\omega^{\beta+2}$-behaviour predicted in [7]. Devaty and Sievers [13] have discussed the quantum theory for absorption within the framework of effective-medium theory [2, 3], and have pointed out some other errors in [7]. Several authors [14-16] have reported calculations of the absorption coefficient based upon elaborate numerical techniques for determining a self-consistent effective potential. These calculations are all confined to exactly spherical geometries with no disorder, and are therefore relevant to the case of ballistic electron motion rather than the diffusive case considered here.

We will derive a new relation for the classical absorption coefficient in section 2 , before discussing the quantum mechanical calculation and the semiclassical approximation in section 3. Section 4 discusses some specific geometries for which we can calculate the absorption coefficient explicitly. Section 5 considers the effects of roughness on the surface of the particles, and section 6 discusses some directions for further research into this topic.

\section{Classical calculation of the absorption coefficient}

The absorption of radiation is usually described by an extinction coefficient $\gamma(\omega)$, which is defined as the fractional loss of intensity per unit length of sample, divided by the volume fraction $\mathcal{F}$ occupied by the particles. We will express our results in terms of the rate of absorption of energy $\langle\mathrm{d} E / \mathrm{d} t\rangle$ within a single particle. If the amplitude of the electric field is $\mathcal{E}_{0}$, the intensity of the radiation is $I=\frac{1}{2} \epsilon_{0} \mathcal{E}_{0}^{2}$, and the relationship between $\gamma$ and $\langle\mathrm{d} E / \mathrm{d} t\rangle$ is therefore

$$
\gamma=\frac{2}{V \epsilon_{0} c \mathcal{E}_{0}^{2}}\left\langle\frac{\mathrm{d} E}{\mathrm{~d} t}\right\rangle
$$

where $V$ is the volume of a single particle. In this paper we will define the absorption coefficient $\alpha(\omega)$ as the rate of absorption of energy for a single particle, divided by the electric field intensity:

$$
\alpha(\omega)=\frac{1}{\mathcal{E}_{0}^{2}}\left\langle\frac{\mathrm{d} E}{\mathrm{~d} t}\right\rangle .
$$


Classically, the rate of absorption of energy is determined by the rate of Ohmic heating within the particle, due to a current density $\operatorname{Re}[\boldsymbol{j}(\boldsymbol{r}, \omega) \exp (\mathrm{i} \omega t)]$ :

$$
\alpha(\omega)=\frac{1}{2 \sigma_{0} \mathcal{E}_{0}^{2}} \int \mathrm{d} \boldsymbol{r}|\boldsymbol{j}|^{2}
$$

where $\sigma_{0}$ is the DC conductivity of the metal, and in this and subsequent expressions the integral is over the volume of the particle.

At frequencies very small compared to the plasma frequency, the current density $j$ can be determined as follows. In a static electric field, a polarization charge is developed which creates a supplementary electric field which exactly cancels the externally applied field within the metal. The polarization charge has density $\rho(\boldsymbol{r})$; in three-dimensional metallic samples this charge is concentrated on the surface of the metal, where it has density per unit area $q(\boldsymbol{r})$. The calculation of the polarization charge distribution is a difficult problem in electrostatics; exact solutions are known for some simple geometries [20].

The current density $j$ is determined by the requirement that it generates the correct screening charge density $\rho$ :

$$
\frac{\partial \rho}{\partial t}+\nabla \cdot j=0
$$

It is driven by a potential energy $U_{\mathrm{dyn}}$ :

$$
j=\frac{\sigma_{0}}{e} \nabla U_{\mathrm{dyn}} .
$$

Equations (2.4) and (2.5) imply that

$$
\nabla^{2} U_{\mathrm{dyn}}=-\frac{\mathrm{i} e \omega}{\sigma_{0}} \rho .
$$

In order to determine $U_{\mathrm{dyn}}$ in terms of the screening charge density $\rho$, we write

$$
U_{\mathrm{dyn}}(\boldsymbol{r}, \omega)=\frac{\mathrm{i} e \omega}{\sigma_{0}} \int \mathrm{d} \boldsymbol{r}^{\prime} G\left(\boldsymbol{r}, \boldsymbol{r}^{\prime}\right) \rho\left(\boldsymbol{r}^{\prime}\right)
$$

where $G\left(\boldsymbol{r}, \boldsymbol{r}^{\prime}\right)$ is a Green's function satisfying $\nabla^{2} G=-\delta\left(\boldsymbol{r}-\boldsymbol{r}^{\prime}\right)$, with the boundary condition $\boldsymbol{n} \cdot \nabla G=0$, where $\boldsymbol{n}$ is a vector normal to the boundary, representing the fact that there is no current across the boundary of the particle. This Green's function is

$$
G\left(\boldsymbol{r}, \boldsymbol{r}^{\prime}\right)=\sum_{n} \frac{\chi_{n}^{*}(\boldsymbol{r}) \chi_{n}\left(\boldsymbol{r}^{\prime}\right)}{k_{n}^{2}}
$$

where the $\chi_{n}(\boldsymbol{r})$ and $k_{n}^{2}$ are orthonormal eigenfunctions and eigenvalues of the Laplacian

$$
\left(\nabla^{2}+k_{n}^{2}\right) \chi_{n}(\boldsymbol{r})=0
$$

with the boundary condition $n \cdot \nabla \chi_{n}=0$. Returning to the evaluation of (2.3), and using the fact that $\boldsymbol{j} \cdot \boldsymbol{n}=0$ on the boundary, we find

$$
\begin{aligned}
& \alpha(\omega)=\frac{\sigma_{0}}{2 e^{2} \mathcal{E}_{0}^{2}} \int \mathrm{d} \boldsymbol{r}\left|\nabla U_{\mathrm{dyn}}\right|^{2} \\
& \quad=-\frac{\sigma_{0}}{2 e^{2} \mathcal{E}_{0}^{2}} \int \mathrm{d} \boldsymbol{r} U_{\mathrm{dyn}}^{*} \nabla^{2} U_{\mathrm{dyn}}=\frac{\mathrm{i} \omega}{2 e \mathcal{E}_{0}^{2}} \int \mathrm{d} \boldsymbol{r} U_{\mathrm{dyn}}^{*}(\boldsymbol{r}, \omega) \rho(\boldsymbol{r}) .
\end{aligned}
$$

Substituting the results of (2.7) and (2.8), the absorption coefficient is therefore

$$
\alpha(\omega)=\frac{\omega^{2}}{2 \sigma_{0} \mathcal{E}_{0}^{2}} \sum_{n} \frac{1}{k_{n}^{2}}\left|\int \mathrm{d} \boldsymbol{r} \chi_{n}(\boldsymbol{r}) \rho(\boldsymbol{r})\right|^{2} .
$$

Equation (2.11) gives the classical absorption coefficient for frequencies small compared to the plasma frequency in terms of the static screening charge density $\rho(\boldsymbol{r})$. 


\section{Semiclassical calculation of absorption coefficient}

In the present section it will be shown how the absorption coefficient $\alpha(\omega)$ may be calculated starting from a microscopic quantum mechanical point of view and subsequent semiclassical approximation. When the particle is subjected to a time-dependent external electric field $\operatorname{Re}\left[\mathcal{E}_{0} \mathrm{e}^{\mathrm{i} \omega t}\right]$, the charges will rearrange in order to screen out the external electric field. We will assume that the screening is governed by an effective one-body potential $U_{\text {eff }}(\boldsymbol{r}, \omega)$. In general this effective potential is to be determined self-consistently from the many-body Schrödinger equation. It will be a function of the frequency $\omega$ of the externally applied electric field. We will write it in the form (1.1), where $U_{\text {stat }}(r)$ denotes a static screening potential which binds the screening charge $\rho(\boldsymbol{r})$. If the frequency is small compared to the plasma frequency, it can be determined in the Thomas-Fermi approximation:

$$
U_{\text {stat }}(\boldsymbol{r})=\frac{1}{e}\left(\frac{\partial N}{\partial \mu}\right)^{-1} \rho(\boldsymbol{r})
$$

where $N$ is the integrated density of states per unit volume for the bulk metal, $\rho(\boldsymbol{r})$ is the screening charge density and $\mu$ is the chemical potential (the application of the Thomas-Fermi approximation in solid-state physics is discussed clearly in [21]). This is an excellent approximation when this potential varies sufficiently slowly on the scale of the Fermi wavelength $\lambda_{F}$,

The dynamical contribution $U_{\mathrm{dyn}}(\boldsymbol{r}, \omega)$, on the other hand, is chosen such that it determines the current flow in the interior of the particle building up the screening charge. In section 2 we described how $U_{\mathrm{dyn}}(\boldsymbol{r}, \omega)$ can be obtained as a solution of Poisson's equation. We will see in the following that for large frequencies $\omega>\omega_{c}$, the dynamical part of the effective potential determines the absorption; on the time-scale given by the external frequency, the electrons do not reach the surface and dissipation occurs in the inside of the particle, caused by the current flow which builds up the screening charge. For small frequencies $\omega<\omega_{c}$, on the other hand, the electrons are scattered by the boundary. Since the dynamical potential vanishes linearly with $\omega$, only the Thomas-Fermi contribution remains and determines the absorption. As opposed to the previous case, the absorption takes place near the surface, where the screening potential is largest.

The absorption coefficient $\alpha(\omega)$ may be expressed in terms of the Kubo formula for non-interacting electrons:

$\alpha(\omega)=\frac{\pi}{2} \frac{\omega}{\mathcal{E}_{0}^{2}} \sum_{m n}\left[f\left(E_{n}\right)-f\left(E_{m}\right)\right]\left|\left\langle\psi_{m}\left|\widehat{U}_{\text {eff }}(\boldsymbol{r}, \omega)\right| \psi_{n}\right\rangle\right|^{2} \delta\left(\hbar \omega-E_{m}+E_{n}\right)$

where $f(E)$ is the Fermi function $(1+\exp [\beta(E-\mu)])^{-1}$, with $\beta=1 / k_{B} T$. We will evaluate the Kubo formula using a semiclassical approximation, discussed in [22], which relates the sum over matrix elements to a classical autocorrelation function $\left\langle U_{\mathrm{eff}}^{*}\left(\boldsymbol{r}_{t}, \omega\right) U_{\mathrm{eff}}(\boldsymbol{r}, \omega)\right\rangle_{E}$; here $\boldsymbol{r}_{t}$ is the position reached by a particle initially at $\boldsymbol{r}$ after time $t$, and the angle brackets denote a microcanonical average over electron trajectories at energy $E$ :

$\alpha(\omega)=\frac{\omega}{\mathcal{E}_{0}^{2}} \frac{\pi}{2} \int_{0}^{\infty} \mathrm{d} E[f(E)-f(E+\hbar \omega)] \frac{g(E)}{\pi \hbar} \operatorname{Re} \int_{0}^{\infty} \mathrm{d} t \mathrm{e}^{\mathrm{i} \omega t}\left\langle U_{\text {eff }}^{*}\left(\boldsymbol{r}_{t}, \omega\right) U_{\text {eff }}(\boldsymbol{r}, \omega)\right\rangle_{E}$

where $g(E)$ is the density of states. Provided that both $k_{B} T$ and $\hbar \omega$ are small compared to energy scales over which $g(E)$ and the correlation function differ significantly from their 
values at the Fermi energy, equation (3.3) can be approximated by

$$
\alpha(\omega)=\frac{\omega^{2}}{2 \mathcal{E}_{0}^{2}} g\left(E_{F}\right) \operatorname{Re} \int_{0}^{\infty} \mathrm{d} t \mathrm{e}^{\mathrm{i} \omega t}\left\langle U_{\text {eff }}^{*}\left(\boldsymbol{r}_{t}, \omega\right) U_{\text {eff }}(\boldsymbol{r}, \omega)\right\rangle_{E_{F}} .
$$

In a diffusive system, the autocorrelation function can be expressed in terms of the diffusion propagator $P\left(\boldsymbol{r}, \boldsymbol{r}^{\prime} ; t\right)$. The diffusion propagator satisfies $\left[D \nabla^{2}-\partial_{t}\right] P\left(\boldsymbol{r}, \boldsymbol{r}^{\prime} ; t\right)=0$, where $D$ is the diffusion constant, with initial condition $P\left(\boldsymbol{r}, \boldsymbol{r}^{\prime} ; 0\right)=\delta\left(\boldsymbol{r}-\boldsymbol{r}^{\prime}\right)$ and vanishing normal derivative at the boundary: $\boldsymbol{n} \cdot \nabla P\left(\boldsymbol{r}, \boldsymbol{r}^{\prime} ; t\right)=0$. The latter condition ensures that there is no flux of electrons across the surface of the particle. In terms of the diffusion propagator we obtain for the autocorrelation function

$\left\langle U_{\text {eff }}^{*}\left(\boldsymbol{r}_{t}, \omega\right) U_{\text {eff }}(\boldsymbol{r}, \omega)\right\rangle=\frac{1}{V} \int \mathrm{d} \boldsymbol{r} \int \mathrm{d} \boldsymbol{r}^{\prime} U_{\text {eff }}^{*}(\boldsymbol{r}, \omega) U_{\text {eff }}\left(\boldsymbol{r}^{\prime}, \omega\right) P\left(\boldsymbol{r}, \boldsymbol{r}^{\prime} ; t\right)$.

We can express $P\left(\boldsymbol{r}, \boldsymbol{r}^{\prime} ; t\right)$ in terms of eigenvalues $k_{n}^{2}$ and eigenfunctions $\chi_{n}(\boldsymbol{r})$ of the Laplacian, satisfying (2.10) with vanishing normal derivative at the surface, $\boldsymbol{n} \cdot \boldsymbol{\nabla} \chi_{n}(\boldsymbol{r})=0$ :

$$
P\left(\boldsymbol{r}, \boldsymbol{r}^{\prime} ; t\right)=\sum_{n} \chi_{n}(\boldsymbol{r}) \chi_{n}\left(\boldsymbol{r}^{\prime}\right) \exp \left(-D k_{n}^{2}|t|\right) .
$$

Substitution into equation (3.2) yields

$$
\alpha(\omega)=\frac{\omega^{2}}{2 \mathcal{E}_{0}^{2}} g\left(E_{F}\right) \frac{1}{V} \sum_{n}\left|\int \mathrm{d} \boldsymbol{r} \chi_{n}(\boldsymbol{r}) U_{\mathrm{eff}}(\boldsymbol{r}, \omega)\right|^{2} \frac{D k_{n}^{2}}{\omega^{2}+\left(D k_{n}^{2}\right)^{2}} .
$$

Using the definitions

$$
\begin{aligned}
& \alpha_{n}=\frac{1}{\mathcal{E}_{0}} \int \mathrm{d} \boldsymbol{r} \chi_{n}(\boldsymbol{r}) U_{\text {stat }}(\boldsymbol{r}) \\
& \mathrm{i} \beta_{n}=\frac{1}{\mathcal{E}_{0}} \int \mathrm{d} \boldsymbol{r} \chi_{n}(\boldsymbol{r}) U_{\text {dyn }}(\boldsymbol{r}, \omega)
\end{aligned}
$$

we can write the absorption $\alpha(\omega)$ as a sum of two terms:

$$
\alpha(\omega)=\alpha_{\text {stat }}(\omega)+\alpha_{\text {dyn }}(\omega)
$$

where

$$
\begin{aligned}
\alpha_{\text {stat }}(\omega) & =\frac{\omega^{2}}{2} g\left(E_{F}\right) \frac{1}{V} \sum_{n}\left|\alpha_{n}\right|^{2} \frac{D k_{n}^{2}}{\omega^{2}+\left(D k_{n}^{2}\right)^{2}} \\
\alpha_{\text {dyn }}(\omega) & =\frac{\omega^{2}}{2} g\left(E_{F}\right) \frac{1}{V} \sum_{n}\left|\beta_{n}\right|^{2} \frac{D k_{n}^{2}}{\omega^{2}+\left(D k_{n}^{2}\right)^{2}} .
\end{aligned}
$$

Using equation (2.7) and the Drude relation between the diffusion constant $D$ and the conductivity $\sigma_{0}$,

$$
\sigma_{0}=e^{2}\left(\frac{\partial N}{\partial \mu}\right) D
$$

we find the following relationship between the coefficients $\alpha_{n}$ and $\beta_{n}$ :

$$
\alpha_{n}=\frac{D k_{n}^{2}}{\omega} \beta_{n} .
$$

For small frequencies the term in equation (3.10) proportional to $\left|\alpha_{n}\right|^{2}$ will be dominant, and absorption is a surface effect. For large $\omega$, on the other hand, the term proportional to 
$\left|\beta_{n}\right|^{2}$ dominates and dissipation occurs in the bulk. Combining the static and the dynamic contribution and making use of the relation

$$
g\left(E_{F}\right)=V\left(\frac{\partial N}{\partial \mu}\right)
$$

we find the simple result

$$
\alpha(\omega)=\frac{\omega^{2}}{2} D^{-1}\left(\frac{\partial N}{\partial \mu}\right) \sum_{n} \frac{\left|\alpha_{n}\right|^{2}}{k_{n}^{2}} .
$$

Using the Drude relation (3.11), this is exactly in agreement with the classical expression (2.11). In particular, the absorption coefficient exhibits no structure at the Thouless frequency. We note that our result, equation (3.14), holds irrespective of shape and dimension of the particles.

In conclusion, in the present section we have calculated the absorption coefficient starting from a microscopic quantum mechanical point of view. We have demonstrated that in order to obtain results consistent with classical macroscopic electrodynamics, it is essential to include a dynamical potential $U_{\text {dyn }}$ in addition to the Thomas-Fermi effective potential.

\section{Results for specific geometries}

In the present section we evaluate the absorption coefficient for three cases in which the screening charge density can be obtained from classical electrostatics, namely for a sheet perpendicular to the electric field, for a spherical particle, and for a disc with the electric field in the plane of the disc. In all cases, the Thomas-Fermi potential $U_{\text {stat }}(\boldsymbol{r})$ can be obtained directly from the charge density, and we can use equation (2.10) to evaluate the classical macroscopic absorption coefficient $\alpha(\omega)$.

We have different motivations for considering these three geometries. We discuss the sheet geometry because it is the simplest case, and it will be used for considering the effects of surface roughness in section 5. We discuss the spherical particle in order to show that our approach reproduces the long-established classical expression. And finally we discuss the disc geometry because well controlled experiments are most easily performed for electrons in two-dimensional microstructures. Our result for the absorption coefficient of this geometry appears to be new.

The results of the previous section show that from a microscopical point of view, there are two contributions to the absorption coefficient, $\alpha_{\text {stat }}(\omega)$ and $\alpha_{\text {dyn }}(\omega)$, which become important at small and large frequencies, respectively. For each geometry we will calculate the coefficients $\alpha_{n}$ (the $\beta_{n}$ are then obtained immediately from (3.12)). Inserting these coefficients into (3.10), in figure 1 we plot the two contributions to $\alpha(\omega)$ separately for each of the three geometries.

\subsection{The conducting sheet}

The simplest case is that of a particle in the shape of a sheet of thickness $a$ in a plane lying perpendicular to the electric field; for definiteness we consider the case of a square sheet with sides of length $L \gg a$. In this case classical electrostatics tells us that the surface charge is $q= \pm \epsilon_{0} \mathcal{E}$ on the two surfaces. We first consider how the absorption coefficient can be calculated by elementary arguments, and then see how the result is confirmed using the general formula (3.14). The current flowing through the sheet is $I=L^{2} q \omega$, and the 

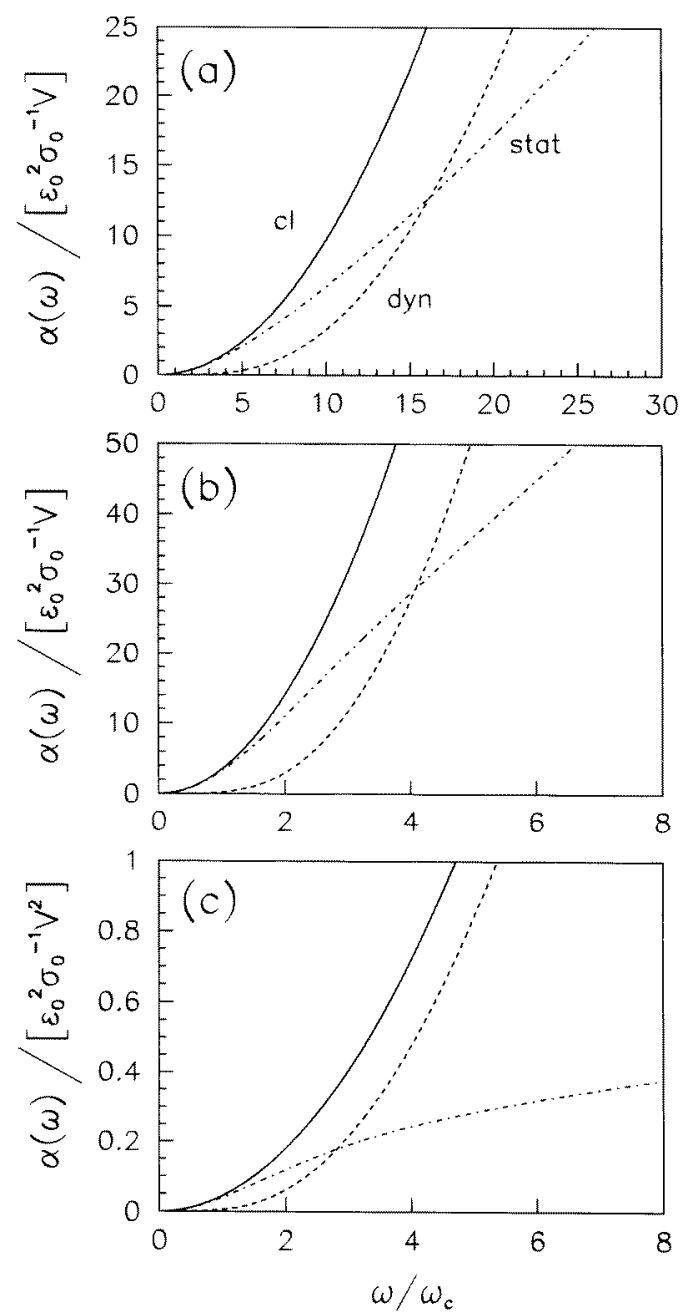

Figure 1. Components of the absorption coefficient for a small metallic particle due to the static potential (stat) and the dynamic potential (dyn), as a function of frequency; the classical absorption according to equation (2.11) is denoted by (cl). We show three cases: (a) the conducting sheet; (b) the spherical particle; and (c) the disc.

resistance of the sheet is $R=a / \sigma_{0} L^{2}$. The absorption coefficient is then

$$
\alpha(\omega)=\frac{1}{2} \frac{I^{2} R}{\mathcal{E}_{0}^{2}}=\frac{\epsilon_{0}^{2} a L^{2} \omega^{2}}{2 \sigma_{0}}
$$

We will now show how this result is recovered from equation (3.14). If the sheet is perpendicular to the $z$-axis, with the surfaces at $z=0$ and $z=a$, the eigenfunctions of the Laplacian are

$$
\chi_{n, p}(\boldsymbol{r})=\sqrt{\frac{2-\delta_{n 0}}{L^{2} a}} \cos (\pi n z / a) \exp \left[\mathrm{i} \boldsymbol{p} \cdot \boldsymbol{r}_{\perp}\right]
$$


where $\boldsymbol{r}_{\perp}=(x, y)$, and the eigenvalues are

$$
k_{n, p}^{2}=\left(\frac{n \pi}{a}\right)^{2}+p^{2} .
$$

Since the surface charge density $q(\boldsymbol{r})$ is constant on each face, the amplitudes $\alpha_{n, p}$ and $\beta_{n, p}$ defined in equation (3.8) vanish unless $\boldsymbol{p}=\mathbf{0}$. Also, since contributions from opposite faces cancel, $\alpha_{n} \equiv \alpha_{n, \mathbf{0}}=0$ and $\beta_{n} \equiv \beta_{n, \mathbf{0}}=0$ unless $n$ is odd. We obtain

$$
\alpha_{n}=\frac{\epsilon_{0} L}{e}\left(\frac{\partial N}{\partial \mu}\right)^{-1} \sqrt{\frac{2}{a}}\left[1-(-1)^{n}\right]
$$

and, using (3.14),

$$
\alpha(\omega)=\frac{\epsilon_{0}^{2} \omega^{2} L^{2} a}{2 \sigma_{0}} \frac{4}{\pi^{2}} \sum_{n=1}^{\infty} \frac{1}{n^{2}}\left[1-(-1)^{n}\right] .
$$

The final summation is equal to $\pi^{2} / 4$, and hence this result agrees with (4.1). The static and dynamical contributions $\alpha_{\text {stat }}$ and $\alpha_{\text {dyn }}$ calculated according to equations (3.10), (3.12) and (4.4) are shown in figure 1(a). The static contribution $\alpha_{\text {stat }}$ is shown as a chain line, while the dynamical contribution $\alpha_{\text {dyn }}$ is shown as a dashed line. The two contributions add up to the classical result, equation (4.1), shown as a solid line.

\subsection{The spherical particle}

The surface charge density of a sphere subjected to an external electric field is, from classical electrostatics, $q=3 \epsilon_{0} \mathcal{E}_{0} \cos \theta$ [20], where $\theta$ is the polar angle measured from the direction of the field. If the radius $a$ of the particle is large compared to the Thomas-Fermi screening length $k_{s}^{-1}$, we can approximate the charge density by

$$
\rho(\boldsymbol{r})=3 \epsilon_{0} \mathcal{E}_{0} \cos \theta k_{\mathrm{s}} \exp \left[k_{\mathrm{s}}(r-a)\right]
$$

where $k_{\mathrm{s}}^{2}=\left(e^{2} / \epsilon_{0}\right)(\partial N / \partial \mu)$ [21]. The static part of the effective potential can then be approximated by

$$
U_{\text {stat }}(\mathrm{r}) \simeq \frac{1}{e}\left(\frac{\partial N}{\partial \mu}\right)^{-1} 3 \epsilon_{0} \mathcal{E}_{0} \cos \theta \delta(r-a) .
$$

The corresponding current density $\boldsymbol{j}(\boldsymbol{r})$ which accumulates the charge density $\rho(\boldsymbol{r})$ is easily seen to be uniform, and the dynamical potential is therefore

$$
U_{\mathrm{dyn}}(\boldsymbol{r})=\frac{e}{\sigma_{0}} \boldsymbol{r} \cdot \boldsymbol{j}=\mathrm{i} \omega \frac{3 e \epsilon_{0}}{\sigma_{0}} \mathcal{E}_{0} r \cos \theta .
$$

Using (2.10), we find

$$
\alpha(\omega)=\frac{6 \pi \epsilon_{0}^{2} a^{3} \omega^{2}}{\sigma_{0}} .
$$

This result gives the standard expression [2] for the extinction coefficient $\gamma$ for a spherical particle as derived from classical electrodynamics.

We will now show how this result is recovered from equation (3.14). Since the angular dependence of the potentials is $\sim \cos \theta$, only eigenfunctions of the form $\chi_{n}(r)=R_{n}(r) \cos \theta$ contribute to the sums in equation (3.10). Up to a normalization constant, the radial eigenfunctions $R_{n}(r)$ are given by $j_{1}\left(k_{n} r\right)$, the first-order spherical Bessel function. The 
appropriate boundary conditions are enforced by requiring that $\partial j_{1}\left(k_{n} r\right) / \partial r=0$ for $r=a$. Using the definitions and results in [23, 24], we obtain the integral

$$
\int_{0}^{a} \mathrm{~d} r r^{2} j_{1}^{2}\left(k_{n} r\right)=\frac{a}{2} \frac{\left(k_{n}^{2} a^{2}-2\right)}{k_{n}^{2}} j_{1}^{2}\left(k_{n} a\right)
$$

which can be used to normalize the eigenfunctions; we then find

$$
\alpha_{n}=\frac{1}{e}\left(\frac{\partial N}{\partial \mu}\right)^{-1} 3 \epsilon_{0} \sqrt{\frac{8 \pi}{3}} \frac{k_{n} a^{3 / 2}}{\sqrt{\left(k_{n} a\right)^{2}-2}} .
$$

Figure 1(b) shows the two contributions $\alpha_{\text {stat }}$ and $\alpha_{\text {dyn }}$ towards the absorption coefficient (3.10), where the coefficients $\beta_{n}$ were obtained using (3.12). In the appendix we derive a sum rule for the zeros $k_{n}$ of $\partial j_{1}\left(k_{n} r\right) / \partial r$, which can be used to verify that the two contributions add up to the classical result, equation (4.9).

\subsection{The conducting disc}

Finally, we discuss the case of a two-dimensional disc. Here, the screening charge density is distributed across the surface of the disc, with density [20]

$$
\rho(\boldsymbol{r})=\frac{4 \epsilon_{0} \mathcal{E}_{0}}{\pi} \frac{r \cos \phi}{\sqrt{a^{2}-r^{2}}}
$$

where $a$ is the radius of the disc. The charge density is therefore slowly varying on the scale of the Fermi wavelength, except for the singularity at the edge of the disc; this suggests that the results of applying the Thomas-Fermi approximation are likely to be very accurate in this case. For two-dimensional discs subjected to an external electric field, the static potential is therefore given by

$$
U_{\text {stat }}(\boldsymbol{r})=\frac{1}{e}\left(\frac{\partial N}{\partial \mu}\right)^{-1} \frac{4 \epsilon_{0} \mathcal{E}_{0}}{\pi} \frac{r \cos \phi}{\sqrt{a^{2}-r^{2}}} .
$$

The dynamical potential $U_{\text {dyn }}$ satisfies Poisson's equation in the form (2.6). If the charge density $\rho$ were a radially symmetric function $g(r)$, the corresponding radially symmetric solution $f(r)$ of Poisson's equation could be found immediately by integrating twice. In equation (4.13) the charge density may be viewed as a dipolar distribution resulting from the infinitesimal displacement of two radially symmetric distributions $g(r)$. The corresponding solution of Poisson's equation is then a dipolar distribution formed from the radially symmetric functions $f(r)$. Following this approach, and applying the appropriate boundary conditions, we find the solution

$$
U_{\mathrm{dyn}}(r, \omega)=-\frac{\mathrm{i} e \omega}{\sigma_{0}} \frac{4 \epsilon_{0} \mathcal{E}_{0}}{\pi} \frac{1}{3}\left[\frac{\left(a^{2}-r^{2}\right)^{3 / 2}}{r}-\frac{a}{r}\left(a^{2}+r^{2}\right)\right] \cos \phi .
$$

Using equation (2.10), we find

$$
\alpha(\omega)=\frac{34}{9 \pi} \frac{\epsilon_{0} a^{4} \omega^{2}}{\sigma_{0}} .
$$

We conclude by determining the coefficients $\alpha_{n}$ for the disc geometry. The eigenfunctions of the Laplacian are $\chi_{n}(r)=R_{n}(r) \cos \phi$, where the radial functions $R_{n}(r)$ are proportional 
to the first-order Bessel function $J_{1}\left(k_{n} r\right)$. The boundary conditions are $\partial J_{1}\left(k_{n} r\right) / \partial r=0$ for $r=a$. Using results in [23, 24] we obtain the integral identities

$$
\begin{aligned}
& \int_{0}^{a} \mathrm{~d} r \frac{r^{2}}{\sqrt{a^{2}-r^{2}}} J_{1}\left(k_{n} r\right)=a^{2} \sqrt{\frac{\pi}{2 k_{n} a}} J_{3 / 2}\left(k_{n} a\right)=a^{2} j_{1}\left(k_{n} a\right) \\
& \int_{0}^{a} \mathrm{~d} r r J_{1}^{2}\left(k_{n} r\right)=\frac{1}{2} \frac{\left(k_{n}^{2} a^{2}-1\right)}{k_{n}^{2}} J_{1}^{2}\left(k_{n} a\right)
\end{aligned}
$$

which are used to calculate the integrals in equation (2.11) and to normalize the eigenfunctions; we find

$$
\alpha_{n}=\frac{4 \epsilon_{0}}{e}\left(\frac{\partial N}{\partial \mu}\right)^{-1} \sqrt{\frac{2}{\pi}} \frac{k_{n} a^{2}}{\sqrt{\left(k_{n} a\right)^{2}-1}} \frac{j_{1}\left(k_{n} a\right)}{J_{1}\left(k_{n} a\right)} .
$$

The resulting contributions to the absorption coefficient are shown in figure 1(c). Again, we show the Thomas-Fermi and the dynamical contributions separately (chain and dashed lines, respectively). The contributions add up to $\alpha(\omega)$ as given in equation (4.15), shown as a solid line.

Comparing the two-dimensional case, equation (4.15), with the three-dimensional cases, equations (4.1) and (4.9), we observe the following. In three dimensions, $\alpha(\omega)$ is proportional to the volume $V$ of the particle. In two dimensions, on the other hand, one has $\alpha(\omega) \sim V^{2}$.

\section{The effects of surface roughness}

In this section we discuss the effects of roughness of the particle surface on the absorption coefficient. Surface roughness alters the distribution of surface charge density $q(\boldsymbol{r})$ : the magnitude of the charge density is decreased within pits in the surface, and increased on prominences. We will calculate the effect of a randomly fluctuating contribution to the surface charge density; we assume that the particle surface is a small deformation of a smooth surface for which the surface charge density is $q_{0}$, and write the total surface charge density as

$$
q(\boldsymbol{r})=q_{0}(\boldsymbol{r})+\delta q(\boldsymbol{r})
$$

where $\delta q(\boldsymbol{r})$ is a small random fluctuation. For simplicity we will consider the slab geometry, discussed in section 4.1 , and assume that the perturbation $\delta q$ is non-zero on only one face of the slab, at $z=a / 2$, so that $\delta q$ is a function of the coordinate vector $\boldsymbol{r}_{\perp}=(x, y)$. We write $(3.14)$ as

$$
\alpha(\omega)=\frac{\omega^{2}}{2 \sigma_{0}} \sum_{n=0}^{\infty} \sum_{p} \frac{\left|a_{n, p}\right|^{2}}{k_{n, p}^{2}} \equiv \frac{\omega^{2}}{2 \sigma_{0}} S
$$

with $a_{n, p}=e(\partial N / \partial \mu) \alpha_{n, p}$, and the sum over $\boldsymbol{p}$ is over a lattice of points with density $1 /(2 \pi L)^{2}$. The sum $S$ now includes contributions from eigenfunctions with $\boldsymbol{p} \neq \mathbf{0}$. The additional contribution to the coefficient $a_{n, p}$ is

$$
\delta a_{n, \boldsymbol{p}}=\frac{1}{\mathcal{E}_{0}} \sqrt{\frac{2}{a L^{2}}} \int \mathrm{d} \boldsymbol{r}_{\perp} \delta q\left(\boldsymbol{r}_{\perp}\right) \exp \left(\mathrm{i} \boldsymbol{p} \cdot \boldsymbol{r}_{\perp}\right)
$$


so the mean value of $\left|\delta a_{n, p}\right|^{2}$ is given by a Fourier transform of the correlation function of $\delta q$ :

$$
\begin{aligned}
& \left\langle\left|\delta a_{n, \boldsymbol{p}}\right|^{2}\right\rangle=\frac{2}{\mathcal{E}_{0}^{2} a} \int \mathrm{d} \boldsymbol{r}_{\perp} C_{\delta q}\left(\boldsymbol{r}_{\perp}\right) \exp \left(\mathrm{i} \boldsymbol{p} \cdot \boldsymbol{r}_{\perp}\right) \equiv \frac{2}{\mathcal{E}_{0}^{2} a} \widetilde{C}_{\delta q}(\boldsymbol{p}) \\
& C_{\delta q}\left(\boldsymbol{r}_{\perp}\right)=\frac{1}{L^{2}} \int \mathrm{d} \boldsymbol{r}_{\perp}^{\prime} \delta q\left(\boldsymbol{r}_{\perp}+\boldsymbol{r}_{\perp}^{\prime}\right) \delta q\left(\boldsymbol{r}_{\perp}^{\prime}\right) .
\end{aligned}
$$

The contribution to the sum $S$ in (5.2) which is linear in $\delta q$ has mean value zero, so the additional contribution to the mean value of the sum from the surface roughness is

$$
\delta S=\frac{2}{\mathcal{E}_{0}^{2} a} \sum_{n=0}^{\infty} \sum_{\boldsymbol{p}} \frac{\widetilde{C}_{\delta q}(\boldsymbol{p})}{\boldsymbol{p}^{2}+n^{2} \pi^{2} / a^{2}} \sim \frac{2\left\langle\delta q^{2}\right\rangle \delta R^{2}}{\mathcal{E}_{0}^{2} a} \sum_{n=0}^{\infty} \sum_{|p|<\delta R^{-1}} \frac{1}{\boldsymbol{p}^{2}+(n \pi / a)^{2}}
$$

where in the second line we have approximated $\widetilde{C}_{\delta q}(p)$ by a function which takes the value $\left\langle\delta q^{2}\right\rangle$ for $|\boldsymbol{p}|<\delta R^{-1}$ and zero elsewhere. Noting that $S \sim a L^{2}\left(q_{0} / \mathcal{E}_{0}\right)^{2}$, we find

$$
\begin{aligned}
\frac{\delta S}{S} \sim \frac{\left\langle\delta q^{2}\right\rangle}{q_{0}^{2}} & \frac{\delta R^{2}}{a^{2} L^{2}} \sum_{n=0}^{\infty} \sum_{|p|<\delta R^{-1}} \frac{1}{p^{2}+(n \pi / a)^{2}} \\
& \sim \frac{\left\langle\delta q^{2}\right\rangle}{q_{0}^{2}} \frac{\delta R^{2}}{a^{2}} \sum_{n=0}^{\infty} \int_{L^{-1}}^{\delta R^{-1}} \mathrm{~d} p \frac{p}{p^{2}+n^{2} \pi^{2} / a^{2}} \widetilde{C}_{\delta q}(p) \\
& \sim \frac{\left\langle\delta q^{2}\right\rangle}{q_{0}^{2}} \frac{\delta R^{2}}{a^{2}}[\log (L)-\log (\delta R)]
\end{aligned}
$$

where in the second equality we have assumed that the statistics of the charge fluctuations is isotropic. We consider the limiting case where the charge fluctuations induced by the surface roughness are small compared to $q_{0}$, and the correlation length $\delta R$ is small compared to $a$. Equation (5.6) then shows that the effect of surface roughness will be small, except in the limit $L \rightarrow \infty$, where the logarithm diverges. If we consider this calculation for a slab geometry as a simplified model for a particle of compact shape, then it is appropriate to regard the ratio $L / a$ as fixed, and the relative effect of surface roughness decreases as $\delta R / a \rightarrow 0$. A more refined model, taking account of how electrostatics influences the correlation function of the surface charge distribution, would be required to give a finite result for the limit $L \rightarrow \infty$ with other quantities fixed; we will not pursue this further.

\section{Concluding remarks}

In this paper we have shown how the absorption coefficient of a small metal particle is determined by the distribution of static polarization charge density. We find that classical and quantum mechanical approaches give the same expressions, and that the quantum mechanical expression is valid both above and below the Thouless frequency $\omega_{c}$. The quantum mechanical expression can be interpreted as consisting of two parts, arising from the static and dynamic parts of the effective one-body potential which is produced in response to the externally applied electric field. The static contribution is dominant below $\omega_{c}$, and the dynamic contribution dominates above $\omega_{c}$, but remarkably the two contributions sum to the classical expression for all frequencies.

We can give explicit formulae for the absorption coefficient in cases where the polarization charge density has been determined by classical electrostatics; we considered spherical particles and discs, and showed that in the former case the result reduces to a known result. We introduced a model for surface roughness, which gives a small contribution 
for particles with a compact shape. The model for surface roughness gives a divergent correction for a slab geometry with aspect ratio $L / a \rightarrow \infty$; a realistic theory for the relationship between surface topography and induced charge density would be required to eliminate this divergence.

The results discussed in this paper contrast in several ways with those for ballistic electron motion, discussed in $[17,18]$. In the latter case there is no requirement for a dynamic potential, because there is no resistance to the movement of the screening charges. Unlike in the diffusive case discussed here, there are important effects at the frequency $\omega_{c}=v_{F} / a$, and the frequency dependence of the absorption coefficient depends upon the dimensionality of the sample. In the ballistic case it was also found that the absorption coefficient can be greatly enhanced by surface roughness in the three-dimensional case.

\section{Acknowledgments}

We are grateful to the organizers of the programme 'Quantum Chaos and Mesoscopic Physics' for inviting us to the Institute for Theoretical Physics (ITP); this program was supported by the National Science Foundation (NSF), grant number 94-07194. The visit of BM was funded by the Max Planck Institute for Physics of Complex Systems, Dresden; BM would like to thank Professor Dr P Fulde for his kind interest, his encouragement, and for making the visit to the ITP possible. The work of MW was supported by research grants from the EPSRC, reference GR/H94337 and GR/L02302, in addition to the generous support of the NSF.

\section{Appendix}

In this appendix the sum

$$
\sum_{n=1}^{\infty} \frac{1}{k_{n}^{2} a^{2}-2}
$$

will be evaluated using contour integration. We define a meromorphic function $f(z)$ with poles at $k_{n} a$ and residues $\left[k_{n}^{2} a^{2}-2\right]^{-1}$. Using $\partial j_{1}\left(k_{n} r\right) / \partial r=0$ at $r=a$, we have

$$
f(z)=\frac{\cos (z)}{\left(z^{2}-2\right) \sin (z)+2 z \cos (z)} \frac{z^{2}}{z^{2}-2} .
$$

This function exhibits poles at $z= \pm k_{n} a$ with the desired residues. In addition to these poles, $f(z)$ has poles at $z= \pm \sqrt{2}$ with residues $1 / 4$ and at $z=0$ with residue $-3 / 2$. Integrating $f(z)$ along the contour enclosing these poles, we obtain

$$
\int_{C} \frac{\mathrm{d} z}{2 \pi \mathrm{i}} f(z)=2 \sum_{n=1}^{\infty} \frac{1}{k_{n}^{2} a^{2}-2}+\frac{1}{2}-\frac{3}{2} .
$$

The contour integral vanishes and therefore

$$
\sum_{n=1}^{\infty} \frac{1}{k_{n}^{2} a^{2}-2}=\frac{1}{2}
$$

Substituting this result into (3.14) and using (4.11), one recovers (4.9). 


\section{References}

[1] Mie G 1908 Ann. Phys., Lpz. 25377

[2] Garnett J C M 1904 Phil. Trans. R. Soc. 203385

[3] Garnett J C M 1906 Phil. Trans. R. Soc. 205237

[4] Perenboom J A A J, Wyder P and Meier F 1981 Phys. Rep. 78173

[5] Carr G L, Perkowitz S and Tanner D B 1985 Infrared and Millimeter Waves vol 13, ed K J Button (New York: Academic) p 169

[6] Halperin W P 1986 Rev. Mod. Phys. 58533

[7] Gorkov L P and Eliashberg G M 1965 Zh. Eksp. Teor. Fiz. 481407 (Engl. Transl. 1965 Sov. Phys.-JETP 21 940)

[8] Porter C E (ed) 1965 Statistical Properties of Spectra: Fluctuations (New York: Academic)

[9] Strässler S, Rice M J and Wyder P 1972 Phys. Rev. B 62575

[10] Rice M J, Strässler S and Wyder P 1973 Phys. Rev. B 8474

[11] Efetov K B 1996 Phys. Rev. Lett. 761908

[12] Sivan U and Imry Y 1987 Phys. Rev. B 356074

[13] Devaty R P and Sievers A J 1980 Phys. Rev. B 222123

[14] Beck D E 1987 Phys. Rev. B 357325

[15] Serra Ll, Garcias F, Barranco M, Navarro J, Balbás L C, Rubio A and Mãnanes A 1989 J. Phys.: Condens. Matter 110391

[16] Hache F and Ricard D 1989 J. Phys.: Condens. Matter 18035

[17] Austin E J and Wilkinson M 1993 J. Phys.: Condens. Matter 58461

[18] Wilkinson M and Austin E J 1994 J. Phys.: Condens. Matter 64153

[19] Thouless D J 1974 Phys. Rep. 1393

[20] Landau L D and Lifshitz E M 1959 Electrodynamics of Continuous Media (Oxford: Pergamon)

[21] Ashcroft N W and Mermin N D 1976 Solid State Physics (Philadelphia, PA: Saunders College)

[22] Wilkinson M 1987 J. Phys. A: Math. Gen. 202415

[23] Abramowitz M and Stegun I (ed) 1965 Handbook of Mathematical Functions (New York: Dover)

[24] Gradshteyn I S and Ryzhik I M (ed) 1981 Table of Integrals, Series and Products (London: Academic) 